\title{
Manifestação da fadiga vocal em docentes de metodologia ativa versus tradicional durante as aulas remotas devido a Covid-19
}

\author{
Vocal fatigue manifestation in professors of active versus traditional teaching methods during \\ remote classes due to Covid-19 \\ Manifestación de fatiga vocal en profesores de metodología activa versus tradicional de enseñanza \\ durante clases remotas debido a Covid-19
}

Recebido: 08/11/2021 | Revisado: 23/11/2021 | Aceito: 24/11/2021 | Publicado: 06/12/2021

\author{
Carla Santana \\ ORCID: https://orcid.org/0000-0002-9354-911X \\ Universidade Federal de Sergipe, Brasil \\ E-mail: carlasantanacarvalho@academico.ufs.br \\ Caroline Oliveira dos Santos \\ ORCID: https://orcid.org/0000-0002-7097-7234 \\ Universidade Federal de Sergipe, Brasil \\ E-mail: carolineoliveira@ academico.ufs.br \\ Aline Ferreira de Brito Mota \\ ORCID: https://orcid.org/0000-0001-5070-4581 \\ Universidade Federal de Sergipe, Brasil \\ E-mail alinebrito@academico.ufs.br \\ Ariane Damasceno Pellicani \\ ORCID: https://orcid.org/0000-0003-0390-9175 \\ Universidade Federal de Sergipe, Brasil \\ E-mail: adpellicani@academico.ufs.br
}

\section{Resumo}

Objetivo: comparar a manifestação da fadiga vocal em professores universitários de metodologia ativa e tradicional no período de aulas remotas devido a pandemia da Covid-19. Métodos: Participaram do estudo 106 professores universitários de uma instituição federal de ensino, que foram agrupados conforme a metodologia utilizada. O grupo de metodologia tradicional (MT) foi composto por 59 professores (31 homens, 28 mulheres), com média de idade de 44,07 $\pm 9,12 \mathrm{O}$ grupo de metodologia ativa (MA) foi composto por 47 professores (20 homens, 27 mulheres), com média de idade de 42,89 \$9,08. Foram utilizados os protocolos: Índice de Fadiga Vocal (IFV), Índice de Triagem do Distúrbio de Voz (ITDV) e anamnese estruturada. A análise envolveu a comparação entre os grupos e comparação entre os gêneros de cada grupo. Resultados: O IFV não apresentou diferença estatística em nenhum dos seus fatores, entretanto, com exceção do fator de restrição vocal, todos os outros parâmetros do teste estiveram acima dos valores de corte. O ITDV e a carga horária de aulas remotas também não apresentaram diferença estatisticamente significante entre os grupos. Mulheres apresentaram valores do IFV superiores a homens, principalmente para metodologia ativa de ensino. Conclusão: Os professores universitários apresentaram manifestações de fadiga vocal, limitação vocal, desconforto físico associado ao uso da voz e dificuldade na recuperação após o repouso vocal, independentemente da metodologia de ensino utilizada.

Palavras-chave: Voz; Fadiga; Docente; Educação à distância; Aprendizagem baseada em problemas; Disfonia.

\begin{abstract}
Objective: to compare the manifestation of vocal fatigue in university professors of active and traditional methodology in the period of remote classes due to the Covid-19 pandemic. Methods: The study included 106 university professors from a federal educational institution, who were grouped according to the methodology used. The traditional methodology group (MT) was composed of 59 teachers (31 men, 28 women), with a mean age of $44.07 \pm 9.12$. The active methodology group (AM) was composed of 47 teachers $(20$ men, 27 women), with a mean age of $42.89 \pm 9.08$. The following protocols were used: Vocal Fatigue Index (IFV), Voice Disorder Screening Index (ITDV) and structured anamnesis The analysis involved comparison between groups and comparison between genders in each group. Results: The IFV showed no statistical difference in any of its factors, however, with the exception of the vocal restriction factor, all other test parameters were above the cutoff values. ITDV and the workload of remote classes also did not present a statistically significant difference between the groups. Women had higher IFV values than men, especially for active teaching methodology. Conclusion: University professors presented
\end{abstract}


manifestations of vocal fatigue, vocal limitation physical discomfort associated with voice use and difficulty in recovery after vocal rest, regardless of the teaching methodology used.

Keywords: Voice; Fatigue; University professor; Education; Distance; Problem-based learning; Dysphonia.

\begin{abstract}
Resumen
Objetivo: comparar la manifestación de fatiga vocal en profesores universitarios de metodología activa y tradicional en el período de clases a distancia por la pandemia del Covid-19. Métodos: El estudio incluyó a 106 profesores universitarios de una institución educativa federal, quienes fueron agrupados según la metodología utilizada. El grupo de metodología tradicional (MT) estuvo compuesto por 59 docentes ( 31 hombres, 28 mujeres), con una edad media de 44,07 \pm 9,12 El grupo de metodología activa (AM) estuvo integrado por 47 docentes ( 20 hombres, 27 mujeres), con una edad media de 44,07 $\pm 9,12$ años. edad media de 42,89 $\pm 9,08$. Se utilizaron los siguientes protocolos: índice de fatiga vocal (IFV), índice de detección de trastornos de la voz (ITDV) y anamnesis estructurada. El análisis implicó la comparación entre grupos y la comparación entre géneros en cada grupo. Resultados: La VIF no mostró diferencias estadísticas en ninguno de sus factores, sin embargo, con la excepción del factor de restricción vocal, todos los demás parámetros de la prueba estuvieron por encima de los valores de corte. La ITDV y la carga de trabajo de las clases remotas tampoco presentaron una diferencia estadísticamente significativa entre los grupos. Las mujeres tenían valores de VIF más altos que los hombres, especialmente para la metodología de enseñanza activa. Conclusión: los profesores universitarios presentaron manifestaciones de fatiga vocal, limitación vocal, malestar físico asociado al uso de la voz y dificultad en la recuperación tras el reposo vocal, independientemente de la metodología de enseñanza utilizada.
\end{abstract}

Palabras clave: Voz; Fatiga; Educación a distancia; Docente; Aprendizaje basado en problemas; Disfonía.

\title{
1. Introdução
}

O decreto de pandemia devido ao novo coronavírus (SARS-CoV2), também conhecido como Covid-19, promoveu no mundo a necessidade de medidas de isolamento e distanciamento social que implicaram na mudança na organização do trabalho de vários setores (Ministério da Saúde, 2019.).

Diante disso, vários setores e instituições passaram a executar suas atividades de forma remota, sendo essa, uma possibilidade de manter seu funcionamento durante a pandemia da Covid-19. O trabalho remoto (home office) surge como alternativa emergencial (Bernardo et al., 2020). Esse contexto não foi diferente na Fonoaudiologia, visto que pesquisas recentes em voz têm se dedicado a compreender o uso profissional da voz neste período pandêmico (Nemr et al., 2021), o impacto do uso de máscaras faciais (Ribeiro et al., 2020) e os riscos de alterações vocais em diferentes profissionais que trabalham remotamente (Kenny, 2020; Siqueira et al., 2020) e os desafios da terapia vocal virtual (Cantarella et al., 2020; Castillo-Allendes et al., 2021).

No que diz respeito aos professores, os exercícios de suas atividades laborais também tiveram modificações, suas casas e computadores tornaram-se salas de aula, e foi preciso adaptar as práticas pedagógicas para uma nova modalidade de ensino, o ensino remoto. O novo cenário foi, para muitos, apresentado sem que houvesse um treinamento prévio, gerando estresse e ansiedade (Besser et al., 2020).

O formato presencial da metodologia tradicional proporciona aos docentes alto risco para os distúrbios de voz relacionados ao trabalho. Essa afirmação pode ser confirmada visto o grande número de publicações, nacionais e internacionais, referentes a voz do professor e à busca pela compreensão da complexa realidade do uso vocal na docência, visto que a voz é o principal instrumento de trabalho do professore e que existem múltiplos fatores de risco à saúde vocal (Valente et al., 2015).

O uso prolongado da voz do professor leva a frequentes relatos de fadiga vocal, sendo esta definida como uma adaptação vocal negativa, autorrelatada pelo sujeito e derivada do uso prolongado da voz (Abou-Rafée et al., 2019; Solomon, 2008). A presença de fadiga vocal é frequentemente caracterizada como uma síndrome global identificada por vários sintomas, como aumento da sensação de esforço, desconforto laríngeo, tensão no pescoço e nos ombros, dor no pescoço ou na garganta, perda da flexibilidade e projeção vocal (Abou-Rafée et al., 2019; Gotaas \& Starr, 1993; Ariane Damasceno Pellicani et al., 2015; Solomon, 2008). 
Dentre as razões que ocasionam o desenvolvimento da fadiga vocal neste grupo, encontram-se tanto as condições do ambiente de trabalho quanto a própria atuação do profissional nele. Além disso, soma-se a alta carga horária trabalhada, que faz com que os docentes sejam privados do repouso vocal necessário para recuperar a voz. Verificaram (Laukkanen et al., 2008; A.D. Pellicani et al., 2017) que a utilização da voz por tempo prolongado, principalmente pelos professores, pode ocasionar o aumento da frequência de queixas de cansaço e fadiga vocal que podem resultar no surgimento de disfonias.

As atividades de ensino podem ser desenvolvidas através da metodologia tradicional ou na metodologia ativa de ensino. No método de ensino tradicional, o professor é o único responsável por transmitir conhecimentos para os alunos e, consequentemente, utiliza sua voz demasiadamente. Na metodologia ativa o ensino e a aprendizagem, o professor tem outra função e ganham um formato dialético; nesse formato, o aluno é ativo na sua formação (Paiva et al., 2016) e, assim, os professores diminuem o tempo de exposição vocal nas aulas.

Estudos trazem discussões a respeito de sintomas vocais nos professores das duas metodologias. Os sintomas vocais mais relatados pelos professores da metodologia ativa de ensino são: garganta seca, falha na voz, rouquidão e cansaço ao falar (Bastos, 2019). Já no método tradicional, estão presentes os seguintes sintomas: garganta e tosse seca, pigarro e rouquidão, voz grossa e secreções, falha na voz e tosse com secreção, perda da voz, cansaço ao falar, dor ao engolir ou falar' (Santos, 2019).

Considerando na literatura existe uma lacuna quanto as influências dessas metodologias de ensino na produção vocal do professor, o presente estudo buscou obter informações a respeito da manifestação de fadiga vocal e de um provável distúrbio vocal em professores universitários de metodologia ativa de ensino e compará-los a professores de metodologia tradicional, durante das aulas remotas emergenciais na pandemia da Covid-19.

\section{Metodologia}

Trata-se de um estudo primário, analítico, observacional, transversal, com abordagem quantitativa do tipo descritiva e comparativa, cuja coleta de dados permitiu a aplicação de análise estatística. O estudo obteve aprovação do Comitê de Ética em Pesquisa com seres humanos por meio da Plataforma Brasil, sob o número de CAEE XXX e do Parecer $n^{\circ}$ XXX, no qual toda a coleta de dados ocorreu de forma eletrônica no período de dezembro de 2020 a março de 2021.

\section{Amostra}

Participaram do estudo 106 docentes universitários efetivos atuantes na metodologia ativa ou tradicional de uma universidade federal. Foram incluídos na pesquisa professores do gênero e raça autodeclarada com idade entre de 25 e 65 anos, com carga horária de aula e uso da voz para graduação e/ou pós-graduação.

Não foram aceitos professores remanejados da sala de aula ou que exerciam apenas atividades administrativas no momento da coleta de dados.

A amostra foi dividida em dois grupos: 59 professores atuantes exclusivamente na metodologia tradicional (GMT) e 47 professores atuantes exclusivamente na metodologia ativa (GMA), ambos os grupos foram compostos por números semelhantes. Os professores mantiveram a mesma metodologia aplicada no ensino presencial.

Para o cálculo amostra, inicialmente, foi realizada a aplicação dos protocolos em 15 sujeitos de cada grupo. O p-valor considerado para significância estatística foi de $5 \%$, com probabilidade de erro tipo II de $20 \%$ e poder d e teste de $80 \%$. Assim, o número mínimo para o GMT foi 42 e, para o GMA de 36. No entanto, após os convites para participação na pesquisa, foi obtido um total de 59 indivíduos no GMT e 47 no GMA.

\section{Procedimentos}

Após o aceite de participação, concordância e assinatura do Termo de Consentimento Livre e Esclarecido (TCLE), os professores foram submetidos à coleta de dados. Primeiramente, no intuito de verificar a inclusão na amostra foi aplicado um 
questionário de anamnese para conhecimento dos antecedentes pessoais, situação funcional e ambiente de trabalho no home office

Para verificar a existência de sintomas da fadiga vocal, foi aplicado o Índice de Fadiga Vocal - IFV (Zambon, Moreti, Veis Ribeiro, et al., 2020). Este protocolo investiga a presença da fadiga vocal e sua relação com a restrição vocal, limitação vocal, desconforto físico associado a voz e recuperação vocal após o repouso vocal. É um instrumento adaptado, traduzido e validado para o português brasileiro (Zambon, Moreti, Ribeiro, et al., 2020). Nesta última versão, o protocolo é composto por 17 itens e quatro fatores de análise, sendo eles: cansaço e limitação vocal (IFV1) com índices de corte de 4,50, restrição vocal (IFV2) 3,50, desconforto e voz (IFV 3) com índice de corte de 1,50 e recuperação após o repouso vocal (IFV4) com 8,50 para corte. O valor para indicar a presença de fadiga vocal é de 11,50 pontos (IFV total). Também foi aplicado o Índice de Triagem do Distúrbio de Voz -ITDV (Ghirardi et al., 2013) com o objetivo de verificar a existência de um provável distúrbio de voz na amostra estudada.

\section{Análise dos dados}

Os dados foram tabulados em planilha Excel® e distribuídos conforme os grupos, as variáveis estudadas foram: os quatro fatores do IFV, ITDV e variáveis do questionário de anamnese. Além da análise comparativa entre os grupos pela metodologia, também foi analisada a influência do gênero nas respostas de cada grupo.

Para a análise comparativa para as variáveis ITDV, IFV total e suas categorias foi aplicado o teste de Mann- Whitney. Para a análise das variáveis categóricas de situação funcional e antecedentes pessoais foi aplicado o teste de Chi-quadrado. A análise da regressão múltipla foi aplicada para investigar o grupo de variáveis que impactaram no resultado final do IFV total. Foi aceito valor de $\mathrm{p} \leq 0,05$

\section{Resultados}

O presente estudo obteve a participação de 106 professores universitários, sendo 47 para metodologia ativa (GMA) e 59 para tradicional (GMT). O grupo MA constou de 20 homens e 27 mulheres com média de idade de 42,14 anos $( \pm 7,4$ desvio padrão). O grupo MT constou de 31 homens e 28 mulheres com média de idade de 46,25 anos ( \pm 10,25 desvio padrão).

Foi possível observar que o índice de fadiga vocal (IFV) e ITDV não apresentaram diferença estatisticamente significante (Tabela 1). No entanto, ambos os grupos de professores apresentaram resultados de IFV acima do valor de corte nos fatores 1,3 e 4, referentes a fadiga e limitação vocal, desconforto físico associado à voz e recuperação com repouso vocal, respectivamente. De forma geral, o IFV total de ambos os grupos apresentou valores acima do índice de corte, o que sugere a presença da fadiga vocal nos professores universitários.

Tabela 1: Comparativa do ITDV e Índice de Fadiga Vocal (IFV) entre os grupos de metodologia ativa e tradicional.

\begin{tabular}{|c|c|c|c|c|c|c|c|c|c|c|}
\hline \multirow{2}{*}{ Variáveis } & \multirow{2}{*}{ Valor de corte } & \multicolumn{4}{|c|}{ Tradicional $(\mathbf{n}=59)$} & \multicolumn{4}{|c|}{ Ativo $(n=47)$} & \multirow{2}{*}{ p-valor } \\
\hline & & Média & D.P & Min & Máx & Média & D.P & Min & Máx & \\
\hline ITDV & 5 & 2,81 & 2,28 & 0 & 9 & 2,68 & 2,22 & 0 & 8 & 0,76 \\
\hline IFV total & 11,5 & 17,23 & 7,51 & 3 & 40 & 19,75 & 10,04 & 8 & 46 & 0,21 \\
\hline IFV1 & 4,5 & 6,4 & 5,28 & 0 & 20 & 7,51 & 5,71 & 0 & 22 & 0,29 \\
\hline IFV2 & 3,5 & 2,54 & 2,26 & 0 & 9 & 3,14 & 3,4 & 0 & 12 & 0,81 \\
\hline IFV3 & 1,5 & 2,23 & 3,45 & 0 & 16 & 3,36 & 4,17 & 0 & 16 & 0,18 \\
\hline IFV4 & 8,5 & 5,94 & 4,53 & 0 & 12 & 6,25 & 4,27 & 0 & 12 & 0,79 \\
\hline
\end{tabular}

*Teste Mann-Whitney, $\mathrm{p} \leq 0,05$. ITDV= Índice de Triagem do Distúrbio de Voz. IFV total = fadiga vocal. IFV1= fadiga e limitação vocal. IFV2 = restrição vocal. IFV3= desconforto físico associado a voz. IFV4= recuperação com repouso vocal. Fonte: Autores. 
Foi realizada a análise comparativa entre os gêneros dentro de cada grupo (Tabela 2 e 3). No GMT ambos os gêneros apresentaram valores acima do estabelecido pelo teste, sem diferença estatisticamente significante. No entanto, o gênero feminino apresentou valores absolutos superiores os do gênero masculino.

Tabela 2: Comparativa entre os gêneros para o ITDV e Índice de Fadiga Vocal (IFV) entre os grupos de metodologia tradicional.

\begin{tabular}{|c|c|c|c|c|c|c|c|c|c|c|}
\hline \multirow{2}{*}{ Variáveis } & \multirow{2}{*}{ Valor normalidade } & \multicolumn{4}{|c|}{ Homens $=31$} & \multicolumn{4}{|c|}{ Mulheres=28 } & \multirow{2}{*}{ p-valor } \\
\hline & & Média & D.P & Min & Máx & Média & D.P & Min & Máx & \\
\hline ITDV & 5 & 2,41 & 1,85 & 0 & 6 & 3,25 & 2,64 & 0 & 9 & 0,25 \\
\hline IFV total & 11,5 & 16,25 & 6,45 & 7 & 31 & 18,32 & 8,25 & 3 & 40 & 0,25 \\
\hline IFV1 & 4,5 & 6,19 & 5,09 & 0 & 19 & 6,64 & 5,57 & 0 & 20 & 0,88 \\
\hline IFV2 & 3,5 & 2,38 & 2,33 & 0 & 9 & 2,71 & 2,22 & 0 & 7 & 0,42 \\
\hline IFV3 & 1,5 & 1,64 & 2,12 & 0 & 10 & 2,82 & 4,41 & 0 & 16 & 0,74 \\
\hline IFV4 & 8,5 & 5,96 & 4,56 & 0 & 12 & 5,92 & 4,57 & 0 & 12 & 0,87 \\
\hline
\end{tabular}

*Teste Mann-Whitney, $\mathrm{p} \leq 0,05$. ITDV= Índice de Triagem do Distúrbio de Voz. IFV total = fadiga vocal. IFV1= fadiga e limitação vocal. IFV2 = restrição vocal. IFV3= desconforto físico associado a voz. IFV4= recuperação com repouso vocal. Fonte: Autores.

A Tabela 3 apresenta os resultados na comparação de gênero na metodologia ativa. As mulheres apresentaram diferença estatisticamente significante para o IFV total $(p=0,03)$ e desconforto físico associado a voz $(p=0,02)$.

Tabela 3: Comparativa entre os gêneros para o ITDV e Índice de Fadiga Vocal (IFV) entre os grupos de metodologia ativa.

\begin{tabular}{|c|c|c|c|c|c|c|c|c|c|c|}
\hline \multirow{2}{*}{ Variáveis } & \multirow{2}{*}{ Valor normalidade } & \multicolumn{4}{|c|}{ Homens $=20$} & \multicolumn{4}{|c|}{ Mulheres=27 } & \multirow{2}{*}{ p-valor } \\
\hline & & Média & D.P & Min & Máx & Média & D.P & Min & Máx & \\
\hline ITDV & 5 & 2,25 & 2,02 & 0 & 6 & 3 & 2,35 & 0 & 8 & 0,3 \\
\hline IFV total & 11,5 & 16,15 & 6,45 & 8 & 37 & 22,44 & 11,42 & 9 & 46 & $0,03 *$ \\
\hline IFV1 & 4,5 & 5,75 & 4,47 & 0 & 17 & 8,81 & 6,25 & 0 & 22 & 0,09 \\
\hline IFV2 & 3,5 & 2,65 & 2,47 & 0 & 8 & 3,51 & 3,96 & 0 & 12 & 0,84 \\
\hline IFV3 & 1,5 & 1,85 & 2,88 & 0 & 11 & 4,48 & 4,66 & 0 & 16 & $0,02 *$ \\
\hline IFV4 & 8,5 & 6,1 & 4,55 & 0 & 12 & 6,37 & 4,13 & 0 & 12 & 0,93 \\
\hline
\end{tabular}

*Teste Mann-Whitney, p $\leq 0,05$. ITDV= Índice de Triagem do Distúrbio de Voz. IFV total = fadiga vocal. IFV1= fadiga e limitação vocal. $\mathrm{IFV} 2$ = restrição vocal. IFV3= desconforto físico associado a voz. IFV4= recuperação com repouso vocal. Fonte: Autores.

Na Tabela 4, é possível observar a descrição do histórico de saúde e situação funcional dos professores de ambas as metodologias. 
Tabela 4: Comparativa do histórico de saúde e situação funcional de professores universitários de metodologia tradicional e ativa.

\begin{tabular}{|c|c|c|c|c|}
\hline Variáveis & & $\begin{array}{c}\text { Tradicional }(\mathbf{n}=59) \\
\text { N }(\%)\end{array}$ & $\begin{array}{c}\text { Ativo }(\mathrm{n}=47) \\
\mathbf{N}(\%)\end{array}$ & p-valor \\
\hline \multirow{2}{*}{ Gênero } & Mulheres & $28(47,46)$ & $27(57,45)$ & \multirow{2}{*}{0,3} \\
\hline & Homens & $31(52,54)$ & $20(42,55)$ & \\
\hline \multirow{2}{*}{ Queixa vocal } & Não & $35(59,32)$ & $31(65,96)$ & \multirow{2}{*}{0,48} \\
\hline & Sim & $24(40,68)$ & $16(34,04)$ & \\
\hline \multirow{2}{*}{ Covid 19} & Não & $49(83,05)$ & $31(65,94)$ & \multirow{2}{*}{$0,04 *$} \\
\hline & Sim & $10(16,95)$ & $16(34,04)$ & \\
\hline \multirow{2}{*}{ Problemas de voz anterior } & Não & $33(55,93)$ & $34(72,34)$ & \multirow{2}{*}{0,08} \\
\hline & Sim & $26(44,07)$ & $13(27,66)$ & \\
\hline \multirow{2}{*}{ Voz forte intensidade } & Não & $31(52,54)$ & $23(48,94)$ & \multirow{2}{*}{0,71} \\
\hline & Sim & $28(47,46)$ & $24(51,06)$ & \\
\hline \multirow{2}{*}{ Cansaço vocal } & Não & $36(61,02)$ & $34(72,34)$ & \multirow{2}{*}{0,22} \\
\hline & Sim & $23(38,98)$ & $13(27,66)$ & \\
\hline \multirow{2}{*}{ Problemas na voz } & Não & $54(91,53)$ & $45(95,74)$ & \multirow{2}{*}{0,38} \\
\hline & Sim & $5(8,57)$ & $2(4,26)$ & \\
\hline \multirow{2}{*}{ Cirurgia laringea prévia } & Não & $57(96,61)$ & $44(93,62)$ & \multirow{2}{*}{0,47} \\
\hline & Sim & $2(3,39)$ & $3(6,38)$ & \\
\hline \multirow{2}{*}{ Diagnóstico Otorrinolaringológico } & Não & $51(86,44)$ & $38(80,85)$ & \multirow{2}{*}{0,43} \\
\hline & Sim & $8(13,56)$ & $9(19,15)$ & \\
\hline \multirow{2}{*}{ Lesão laríngea } & Não & $45(76,27)$ & $40(85,11)$ & \multirow{2}{*}{0,25} \\
\hline & Sim & $14(23,73)$ & $7(14,89)$ & \\
\hline \multirow{2}{*}{$\begin{array}{l}\text { Terapia fonoaudiológica } \\
\text { prévia }\end{array}$} & Não & $51(86,44)$ & $46(97,87)$ & \multirow{2}{*}{$0,03 *$} \\
\hline & Sim & $8(13,56)$ & $1(2,13)$ & \\
\hline \multirow{2}{*}{ Remanejamento } & Não & $55(93,22)$ & $45(95,74)$ & \multirow{2}{*}{0,57} \\
\hline & Sim & $4(6,78)$ & $2(4,76)$ & \\
\hline \multirow{2}{*}{ Queixa auditiva } & Não & $52(88,14)$ & $42(89,36)$ & \multirow{2}{*}{0,84} \\
\hline & Sim & $7(11,86)$ & $5(10,64)$ & \\
\hline
\end{tabular}

*Teste Chi-quadrado, $\mathrm{p} \leq 0,05$. Fonte: Autores.

A regressão múltipla possibilitou observar que queixa, cansaço vocal, histórico de cirurgia, tratamento atual com ORL e metodologia ativa contribuíram para o aumento da percepção da fadiga vocal, demonstrado pelo resultado do IFV total (Tabela 5).

Tabela 5: Regressão múltipla para variáveis gênero, queixa, cansaço vocal, histórico de cirurgia, tratamento atual com ORL e metodologia em relação ao IFV total.

\begin{tabular}{|c|c|c|c|c|c|}
\hline \multirow{2}{*}{$\frac{\text { Variáveis }}{\text { Gênero }}$} & \multirow{2}{*}{$\frac{\text { Coeficiente }}{-2,31}$} & \multirow{2}{*}{$\frac{\text { Erro padrão }}{1,41}$} & \multirow{2}{*}{$\frac{\text { p-valor }}{0,1}$} & \multicolumn{2}{|c|}{ IC $95 \%$} \\
\hline & & & & $-5,12$ & 0,49 \\
\hline Queixa & 8,14 & 1,48 & $<0,0001 *$ & 5,19 & 11,09 \\
\hline Cansaço vocal & 9,72 & 2,82 & $0,001 *$ & 4,11 & 15,33 \\
\hline Histórico de cirurgia & 9,63 & 3,35 & $0,005^{*}$ & 2,97 & 16,28 \\
\hline Tratamento ORL atual & $-4,55$ & 1,93 & $0,021 *$ & $-8,4$ & $-0,7$ \\
\hline Metodologia & 3,59 & 1,4 & $0,012 *$ & 0,8 & 6,38 \\
\hline
\end{tabular}

*Regressão Múltipla, R2=0,35, p $\leq 0,05$. Fonte: Autores. 


\section{Discussão}

Dentre a classe conhecida como profissionais da voz, a profissão do professor se enquadra como a mais vulnerável para a ocorrência da disfonia, sujeitos à sobrecarga fonatória e ajustes vocais inadequados (Giannini et al., 2013). O uso prolongado da voz é um dos principais fatores para o aumento do esforço fonatório e, consequentemente, surgimento dos sintomas da fadiga vocal (McCabe \& Titze, 2002), tais como a rouquidão, soprosidade, perda da potência vocal e extensão fonatória, dificuldade para realizar sons agudos, dor e ardor ao falar, odinofagia, secura na laringe e faringe, pigarro e tosse, sendo que tais sintomas tendem a melhorar com o repouso vocal (Nanjundeswaran et al., 2015, 2017; Pellicani et al., 2015).

A análise de regressão deste trabalho possibilitou a observação de que a existência de queixa vocal, associado a percepção de cansaço vocal, histórico de cirurgia, tratamento com otorrinolaringologista no momento atual e a metodologia ativa são fatores que impactam na percepção da fadiga vocal. Com exceção da variável "metodologia ativa", esperava-se que os outros fatores apresentassem relação direta com o aumento da fadiga vocal, visto que a presença de uma lesão laríngea ou mesmo uma disfonia com base comportamental pode levar ao aumento da percepção da fadiga vocal.

Uma das hipóteses do presente estudo era que professores de metodologia tradicional apresentariam maiores queixas quanto a fadiga vocal, visto a necessidade de maior uso vocal nas aulas expositivas. No entanto, ambos os grupos apresentaram resultados superiores aos valores de corte, indicando a fadiga vocal também nos professores de metodologia ativa.

Um estudo (Bastos, 2019) avaliou a sintomatologia vocal de professores universitários de metodologia ativa que atuavam presencialmente, pré pandemia, e observaram queixa de garganta seca, falha na voz, rouquidão e cansaço ao falar. Essas queixas estariam relacionadas a atividades de habilidades, práticas de ensino na comunidade, estágios e seminários, mas não nas atividades de tutorias.

Estudos anteriores ao período pandêmico observaram presença de fadiga e limitação vocal em professores de ensino tradicional, quando avaliados no início e término do ano letivo (Cercal et al., 2019). Valores elevados para desconforto físico associado a voz também são relatados na classe de professores de metodologia tradicional (Cercal et al., 2019; Porto et al., 2021)

Apesar da manifestação da fadiga vocal, a aplicação do IFV permitiu observar que os ambos os grupos conseguem obter recuperação vocal após o repouso, o que é condizente ao relatado na literatura (Cercal et al., 2019; Porto et al., 2021; Zambon, Moreti, Ribeiro, et al., 2020). Um fator importante a ser ressaltado é que ambos os grupos obtiveram médias abaixo ao valor de corte para o Índice de Triagem do Distúrbio de Voz (ITDV), o que sugere a ausência da instalação do distúrbio vocal nesses professores.

No entanto, diferentemente de professores de ensino infantil e médio da rede municipal e estadual, os professores aqui estudados atuavam no ensino universitário, federal, em regime de dedicação exclusiva, com carga horária máxima de sala de aula de $12 \mathrm{~h}$ /semanais, o que os permite ter um período para descanso do uso prolongado da voz.

A ocorrência da fadiga vocal tende a ser maior em mulheres, devido a configuração anátomofuncional das pregas vocais e laringe feminina. O presente estudo também analisou a manifestação da fadiga vocal de acordo com o gênero dos professores de cada grupo, no qual foi observado valores superiores para o IFV total $(\mathrm{p}=0,03)$ e desconforto físico associado a $\operatorname{voz}(p=0,02)$, para o gênero feminino, com diferença estatisticamente significante para o grupo de metodologia ativa.

A necessidade do ensino remoto trouxe uma nova realidade para os professores de metodologia ativa, com necessidade de gravações de vídeos e atividades para o estudo assíncrono e, provavelmente, maior uso vocal. Outro fator que pode ter afetado ambos os grupos, é a mudança na rotina e situação ambiental para o provimento de suas aulas, como a necessidade do uso de telas, fones de ouvido, falar com postura ergonomicamente incorreta, sentado em poltronas, além de toda a tecnologia que pode não ter sido de fácil utilização a todos os professores. 
Configurados no "homeoffice", professores do gênero feminino podem sentir-se ainda mais sobrecarregadas. Um estudo indica (Bridi et al., 2020) que o índice de mulheres que trabalhavam sete dias antes da pandemia, aumentou 15,96\% do trabalho durante a pandemia e que mulheres com filhos têm chance de trabalhar mais vezes na semana. Os autores ainda relatam que, de forma geral, o trabalho remoto realizado por homens e mulheres é desigual, uma vez que as mulheres cuidam dos filhos e assumem atividades domésticas em maior escala, quando comparado aos homens.

Assim, as professoras de metodologia ativa podem ter sentido a sobrecarga geral desse momento de pandemia e demonstrarem maiores queixas de fadiga por meio da voz, visto que as mulheres apresentam maior ocorrência de sintomas vocais, bem como maiores chances de virem a desenvolver maiores queixas de fadiga vocal e presença de alterações vocais (Smith et al., 1998).

Outro fator que não pode ser desconsiderado é que o uso intenso de telas e fones de ouvido podem impactar negativamente no indivíduo. Essa pode ser considerada uma limitação do presente estudo, visto que não foi solicitado ao participante a percepção dessas variáveis na sua nova rotina e, assim, avaliar uma possível influência no resultado da fadiga vocal.

Professores da metodologia ativa apresentaram maior número de casos de Covid-19, o que também pode ter impactado no resultado final. Um estudo realizado por (Lechien et al., 2020) analisou a relação da Covid-19 e a disfonia, encontraram a presença da disfonia em $26,8 \%$ dos sujeitos com estado leve a moderado de Covid-19, sendo esse, um sintoma presente na lista de sintomas do novo coronavírus (2019). Já o estado grave da doença, pode ocasionar afonia em 3,7\% dos pacientes.

\section{Conclusão}

Professores de metodologia ativa e tradicional apresentam fadiga vocal com manifestação de limitação vocal, desconforto físico associado ao uso da voz com recuperação após o repouso vocal. Mulheres de metodologia ativa apresentaram maiores índices quanto comparado a homens. No presente estudo, a presença de queixa vocal, cansaço vocal, histórico de cirurgia, tratamento atual com ORL e a metodologia ativa aumentaram a percepção da fadiga vocal.

\section{Agradecimentos}

Pró-Reitoria de Graduação- PROGRAD/UFS

\section{Referências}

Abou-Rafée, M., Zambon, F., Badaró, F., \& Behlau, M. (2019). Fadiga vocal em professores disfônicos que procuram atendimento fonoaudiológico. CoDAS, 31(3). https://doi.org/10.1590/2317-1782/20182018120

Bastos, J. (2019). Perfil de sinais e sintomas vocais e laríngeos de professores de metodologia ativa. Universidade Federal de Sergipe- Campus Lagarto.

Bernardo, K. A. da S., Maia, F. L., \& Bridi, M. A. (2020). As configurações do trabalho remoto da categoria docente no contexto da pandemia COVID-19. Novos Rumos Sociológicos, 8(14), 8-39. https://doi.org/10.15210/NORUS.V8I14.19908

Besser, A., Lotem, S., \& Zeigler-Hill, V. (2020). Psychological Stress and Vocal Symptoms Among University Professors in Israel: Implications of the Shift to Online Synchronous Teaching During the COVID-19 Pandemic. Journal of Voice. https://doi.org/10.1016/J.JVOICE.2020.05.028

Bridi, M., Uehara Bezerra, G., \& Pilan Zanoni, A. (n.d.). O trabalho remoto e as condições das mulheres no contexto da pandemia COVID-19.

Cantarella, G., Barillari, M. R., Lechien, J. R., \& Pignataro, L. (2020). The Challenge of Virtual Voice Therapy During the COVID-19 Pandemic. Journal of Voice, 35(3), 336-337. https://doi.org/10.1016/J.JVOICE.2020.06.015

Castillo-Allendes, A., Contreras-Ruston, F., Cantor, Lady, Codino, J., Guzman, M., Malebran, C., Manzano, C., Pavez, A., Vaiano, T., Wilder, F., \& Behlau, M. (2021). Terapia Vocal No Contexto Da Pandemia Do Covid-19; Orientações Para A Prática Clínica. Journal of Voice, 35(5), 808.e13. https://doi.org/10.1016/J.JVOICE.2020.08.019

Cercal, G. C. S., de Paula, A. L., Novis, J. M. M., Ribeiro, V. V., \& Leite, A. P. D. (2019). Fadiga vocal em professores universitários no início e ao final do 
ano letivo. CoDAS, 32(1). https://doi.org/10.1590/2317-1782/20192018233

Ghirardi, A. C., Piccolotto Ferreira, L., Pimentel Pinto Giannini, S., \& Dias De Oliveira Latorre, M. D. R. (2013). Screening index for voice disorder (SIVD): Development and validation. Journal of Voice, 27(2), 195-200. https://doi.org/10.1016/j.jvoice.2012.11.004

Giannini, P., Latorre, M., \& Ferreira, L. (2013). Distúrbio de voz relacionado ao trabalho docente: um estudo caso-controle. CoDAS, 25(06), 566-576. https://doi.org/10.1590/S0102-311X2012001100011

Gotaas, C., \& Starr, C. D. (1993). Vocal fatigue among teachers. Folia Phoniatrica, 45(3), 120-129. https://doi.org/10.1159/000266237

Kenny, C. (2020). Dysphonia and Vocal Tract Discomfort While Working From Home During COVID-19. Journal of Voice : Official Journal of the Voice Foundation. https://doi.org/10.1016/J.JVOICE.2020.10.010

Laukkanen, A. M., Ilomäki, I., Leppänen, K., \& Vilkman, E. (2008). Acoustic Measures and Self-reports of Vocal Fatigue by Female Teachers. Journal of Voice, 22(3), 283-289. https://doi.org/10.1016/j.jvoice.2006.10.001

Lechien, J. R., Chiesa-Estomba, C. M., Cabaraux, P., Mat, Q., Huet, K., Harmegnies, B., Horoi, M., Bon, S. D. L., Rodriguez, A., Dequanter, D., Hans, S., Crevier-Buchman, L., Hochet, B., Distinguin, L., Chekkoury-Idrissi, Y., Circiu, M., El Afia, F., Barillari, M. R., Cammaroto, G., ... Saussez, S. (2020). Features of Mild-to-Moderate COVID-19 Patients With Dysphonia. Journal of Voice: Official Journal of the Voice Foundation. https://doi.org/10.1016/J.JVOICE.2020.05.012

McCabe, D. J., \& Titze, I. R. (2002). Chant therapy for treating vocal fatigue among public school teachers: A preliminary study. American Journal of SpeechLanguage Pathology, 11(4), 356-369. https://doi.org/10.1044/1058-0360(2002/040)

Ministério da Saúde (Brasil) O que é COVID-19. Brasília (DF) 2020. https://coronavirus.saude.gov.br/sobre-a-doenca.

Nanjundeswaran, C., Jacobson, B. H., Gartner-Schmidt, J., Verdolini Abbott, K., \& City, J. (2015). Vocal Fatigue Index (VFI): Development and Validation. Journal of Voice, 29, 433-440. https://doi.org/10.1016/j.jvoice.2014.09.012

Nanjundeswaran, C., VanSwearingen, J., \& Abbott, K. V. (2017). Metabolic Mechanisms of Vocal Fatigue. Journal of Voice, 31(3), 378.e1-378.e11. https://doi.org/10.1016/j.jvoice.2016.09.014

Nemr, K., Simões-Zenari, M., De Almeida, V. C., Martins, G. A., \& Saito, I. T. (2021). COVID-19 and the teacher's voice: self-perception and contributions of speech therapy to voice and communication during the pandemic. Clinics, 76. https://doi.org/10.6061/CLINICS/2021/E2641

Paiva, M. R. F., Parente, J. R. F., Brandão, I. R., \& Queiroz, A. H. B. (2016). Metodologias ativas de ensino-aprendizagem: revisão integrativa. sanare Revista de Políticas Públicas, 15(2), 145-153. https://sanare.emnuvens.com.br/sanare/article/view/1049

Pellicani, A.D., Fontes, A. R., Santos, F. F., Pellicani, A. D., \& Aguiar-Ricz, L. N. (2017). Fundamental Frequency and Formants Before and After Prolonged Voice Use in Teachers. Journal of Voice. https://doi.org/10.1016/j.jvoice.2017.04.011

Pellicani, Ariane Damasceno, Ricz, H. M. A., \& Ricz, L. N. A. (2015). Phonatory function after prolonged voice use in Brazilian woman. CoDAS, 27(4), 392399. https://doi.org/10.1590/2317-1782/20152014201

Porto, V. F., Bezerra, T. T., Zambon, F., \& Behlau, M. (2021). Fadiga, esforço e desconforto vocal em professores após atividade letiva. CoDAS, 33(4), 1-8. https://doi.org/10.1590/2317-1782/20202020067

Ribeiro, V. V., Dassie-Leite, A. P., Pereira, E. C., Santos, A. D. N., Martins, P., \& Irineu, R. de A. (2020). Effect of Wearing a Face Mask on Vocal SelfPerception during a Pandemic. Journal of Voice : Official Journal of the Voice Foundation. https://doi.org/10.1016/J.JVOICE.2020.09.006

Santos. (2019). Sinais e sintomas vocais e laríngeos do professor universitário na metodologia tradicional. Universidade Federal de Sergipe- Campus Lagarto.

Siqueira, L. T. D., Santos, A. P. dos, Silva, R. L. F., Moreira, P. A. M., Vitor, J. da S., \& Ribeiro, V. V. (2020). Vocal Self-Perception of Home Office Workers During the COVID-19 Pandemic. Journal of Voice : Official Journal of the Voice Foundation. https://doi.org/10.1016/J.JVOICE.2020.10.016

Smith, E., Kirchner, H. L., Taylor, M., Hoffman, H., \& Lemke, J. H. (1998). Voice problems among teachers: Differences by gender and teaching characteristics. Journal of Voice, 12(3), 328-334. https://doi.org/10.1016/S0892-1997(98)80022-2

Solomon, N. P. (2008). Vocal fatigue and its relation to vocal hyperfunction. International Journal of Speech-Language Pathology, 10(4), 254-266. https://doi.org/10.1080/14417040701730990

Valente, A. L., Silva, Botelho, C., Cândido Da Silva, A. M., Adriana, C., Silva, M., \& Valente, L. (2015). Artigo Distúrbio de voz e fatores associados em professores da rede pública Voice disorder and associated factors among public schools teachers. Rev. Bras. Saúde Ocup, 40(132), 183-195. https://doi.org/10.1590/0303-7657000093814

Zambon, F., Moreti, F., Ribeiro, V. V., Nanjundeswaran, C., \& Behlau, M. (2020). Vocal Fatigue Index: Validation and Cut-off Values of the Brazilian Version. Journal of Voice : Official Journal of the Voice Foundation. https://doi.org/10.1016/J.JVOICE.2020.06.018

Zambon, F., Moreti, F., Veis Ribeiro, V., Nanjundeswaran, C., Behlau, M., Paulo, ao, \& City, J. (2020). Vocal Fatigue Index: Validation and Cut-off Values of the Brazilian Version. https://doi.org/10.1016/j.jvoice.2020.06.018 